\title{
Influência do Aumento do Tempo Inspiratório na Ventilação Pulmonar de Pacientes Submetidos à Ventilação Mecânica na Modalidade Pressão Controlada*
}

\author{
Influence of the Breathing Time Increase in the Pulmonary Ventilation of \\ Patients Submitted to Mechanical Ventilation in Controlled Pressure Mode
}

Datiene Aparecida Diniz Rodrigues Bernal'1, Baldomero Antonio Kato da Silva², Daniel Martins Pereira ${ }^{3}$

\section{RESUMO}

JUSTIFICATIVA E OBJETIVOS: O suporte ventilatório mecânico é uma das principais modalidades de apoio usadas em terapia intensiva. Na modalidade de pressão pré-determinada a pressão máxima é regulada, mas o volume corrente $\left(V_{T}\right)$ é uma função complexa da pressão aplicada e da sua velocidade em alcançar a pressão-alvo, do tempo inspiratório disponível e da resistência à respiração. O objetivo deste trabalho foi avaliar a influência do incremento do tempo inspiratório na ventilação pulmonar.

MÉTODO: O estudo foi realizado na UTI Adulto do Hospital Regional de Mato Grosso do Sul em Campo Grande/MS. Foram incluídos pacientes adultos, de ambos os sexos, com idade entre 16 e 84 anos, submetidos à ventilação mecânica no modo pressão controlada ou

1. Fisioterapeuta Graduada pela Universidade para o Desenvolvimento do Estado e da Região do Pantanal - UNIDERP.

2. Fisioterapeuta Especialista em Fisioterapia Cardiorrespiratória. Mestrando pelo Programa Multi-Institucional de Pós-Graduação em Ciências da Saúde - UnB/UFMS/UFG. Professor do Curso de Fisioterapia da UNIDERP.

3. Fisioterapeuta Especialista em Fisioterapia Cardiorrespiratória. Mestrando pela Pós-Graduação em Saúde e Desenvolvimento da UFMS. Professor do Curso de Fisioterapia da UNIDERP.

*Recebido da Universidade para o Desenvolvimento do Estado e da Região do Pantanal (UNIDERP), Campo Grande, MS

Apresentado em 02 de março de 2006

Aceito para publicação em 13 de abril de 2006

Endereço para correspondência:

Baldomero Antonio Kato da Silva

Rua Gardênia, 129 - Bloco F/202 Bairro Cidade Jardim

79040-570 Campo Grande, MS

Fone: (67) 9221-3038

E-mail: ftbaldock@uol.com.br

(CAssociação de Medicina Intensiva Brasileira, 2006 assistido-controlada. Foi ajustado o tempo inspiratório em $1 \mathrm{seg}$, incrementando em 0,2 seg até o valor limite de 1,6 seg. Foram avaliados o volume-corrente $\left(V_{T}\right)$ e o volume-minuto $\left(V_{E}\right)$ de 13 pacientes nos tempos inspiratórios de 1,0s; $1,2 \mathrm{~s} ; 1,4 \mathrm{~s}$ e 1,6s.

RESULTADOS: Nas médias dos $V_{T}$ e $V_{E}$ observou-se um aumento crescente após o incremento do tempo inspiratório. Não foram encontrados na literatura consultada, dados que correlacionassem o tempo inspiratório com alterações no volume-corrente.

CONCLUSÕES: O incremento do tempo inspiratório na ventilação por controle de pressão pode ter influência na determinação do volume-corrente ofertado ao paciente.

Unitermos: Ventilação Mecânica, Ventilação Pulmonar, Volume Corrente.

\section{SUMMARY}

BACKGROUND AND OBJECTIVES: The mechanical ventilator support is one of the main used modalities of support in intensive therapy. In the modality of predetermined pressure, the maximum pressure is regulated, but the current volume $\left(V_{T}\right)$ is a complex function of the applied pressure and its speed to reach the pressuretarget, of the available breathing time and the resistance to the breath. This paper has as objective to evaluate the influences of the increment of the breathing time in the pulmonary ventilation.

METHODS: The study was carried through in the Adult ICU of the Regional Hospital of Mato Grosso do Sul, located in Campo Grande. They had been enclosed individuals adult, both genders, between 16 and 84 years, submitted to the mechanical ventilation in controlled pressure mode or in controlled-watched mode. The breathing time was adjusted in $1 \mathrm{sec}$, developing in $0.2 \mathrm{sec}$ until the boundary-value of $1.6 \mathrm{sec}$. The tidal 
volume $\left(\mathrm{V}_{\mathrm{T}}\right)$ and the volume minute $\left(\mathrm{V}_{\mathrm{E}}\right)$ had been evaluated of 13 patients in the breathing times of $1 \mathrm{~s} ; 1.2 \mathrm{~s}$; $1.4 \mathrm{~s}$ and $1.6 \mathrm{~s}$.

RESULTS: In the mean of $V_{T}$ and $V_{E}$ an increasing increase was observed after-increment of the breathing time. We did not find in consulted literature, data which correlated the breathing time with alterations in the current volume.

CONCLUSIONS: The increment of the breathing time in the ventilation for pressure control can have influence in the determination of the current volume offered to the patient.

Key Words: Mechanical ventilation, Pulmonary Ventilation, Tidal Volume.

\section{INTRODUÇÃO}

A monitorização de pacientes sob suporte ventilatório é um fator de primordial importância. A partir da integração de dados colhidos dos testes funcionais com a observação clínica contínua, pode-se agir de forma harmônica, prevenindo complicações letais e aumentando as probabilidades de sucesso no tratamento desses pacientes ${ }^{1}$.

O suporte ventilatório mecânico é uma das principais modalidades de apoio usadas em terapia intensiva. Sendo um elemento essencial na reanimação cardiopulmonar, ele pode salvar vidas durante uma variedade de doenças agudas e crônicas, quando a atividade ventilatória estiver deprimida, ou quando o paciente não apresentar condições neuromusculares para ventilar. Além disso, o pulmão é geralmente um dos principais órgãos envolvidos na falência de múltiplos sistemas. Assim, fornecer ventilação adequada constitui um desafio e influencia o prognóstico do pacientes ${ }^{2}$.

Os ventiladores modernos, de alta capacidade, oferecem modalidades ventilatórias de pressão pré-determinada ou com definição da pressão como opções para assistência ventilatória integral ou parcial. Quando a ventilação é iniciada, essas modalidades aplicam e mantém uma quantidade definida de pressão na abertura da via aérea, até que se obtenha um padrão específico de ciclagem ao tempo do suporte pressórico (controle de pressão ou fluxo). A pressão máxima é regulada, mas o volume-corrente é uma função complexa da pressão aplicada e da sua velocidade em alcançar a pressão-alvo, do tempo inspiratório disponível e da resistência à respiração (complacência, resistências inspiratória e expiratória e auto PEEP) $)^{2}$.

$\mathrm{O}$ advento de equipamentos cada vez mais modernos permite ventilar mecanicamente pacientes com doenças mais graves e, conseqüentemente, com maiores possibilidades de êxito ${ }^{3}$.

São de consenso geral que as ventilações mecânicas controladas, independentes do uso da PEEP, promovem profundas alterações no sistema cardiovascular. Estas alterações são provocadas sobretudo pelo aumento da pressão nas vias aéreas, que é transmitida ao espaço interpleural, com conseqüente aumento da pressão intratorácica ${ }^{4}$.

Atualmente aceita-se plenamente que o uso de ventilação mecânica controlada por pressão melhora a pressão arterial de oxigênio $\left(\mathrm{PaO}_{2}\right)$ com menor fração inspirada de oxigênio $\left(\mathrm{FiO}_{2}\right)^{3}$.

Em alguns pacientes a ventilação artificial é uma tarefa por vezes muito difícil, devido a graves distúrbios na relação $V / Q$. Pode haver uma distribuição desigual do volume-corrente, caso a resistência e/ou a complacência variem muito entre diferentes unidades respiratória. Algumas vias aéreas estão superventiladas e seus alvéolos hiper-distendidos, enquanto outras unidades encontram-se hipoventiladas em relação à sua perfusão. Unidades com grande resistência de suas vias de condução demoram mais a serem preenchidas com determinada porção do volume-corrente ${ }^{1}$.

Em pacientes com diminuição da complacência pulmonar a hiperdistensão alveolar pode causar e perpetuar lesões pulmonares ${ }^{5}$.

Essa hiperdistensão tem como principal contribuinte o uso de altas pressões e/ou volumes ocorrendo ao final da inspiração, podendo causar lesão em alvéolos saudáveis em função da distribuição desigual das doenças pulmonares. Baseados em fatores clínicos e experimentais, recomenda-se que para evitar lesões pulmonares induzidas pelo ventilador, o pico de pressão e a pressão de platô devem ser mantidos, respectivamente em valores menores que 40 a $35 \mathrm{cmH}_{2} \mathrm{O}^{6,7}$.

A ventilação por controle de pressão apesar de sua principal desvantagem (volume-corrente variável) tem sido utilizada com grande êxito nos casos mais graves de SARA ${ }^{6}$.

Em pacientes com insuficiência respiratória aguda secundária a SARA onde uma das principais características clínicas é o decréscimo da complacência do sistema respiratório, o uso de volumes correntes tradicionais (10 a $15 \mathrm{~mL} / \mathrm{kg}$ ) resulta em pressão fre- 
qüentemente elevada nas vias aéreas, tendo como reflexo a hiperdistensão nas regiões menos afetadas do pulmão. A ventilação com limitação das pressões nas vias aéreas podem reduzir as lesões pulmonares associadas ao ventilador ${ }^{9}$.

É de grande importância o suporte ventilatório mecânico na terapia intensiva, pois fornece o tratamento ventilatório adequado e influencia no prognóstico do paciente, bem como os benefícios dos modos ventilatórios controlados à pressão são bem conhecidos e discutidos na literatura consultada. Entretanto, observa-se escassez de estudos correlacionando a influência de alterações no tempo inspiratório sobre o volume corrente ofertado aos pacientes.

O objetivo deste trabalho foi avaliar a influência do incremento do tempo inspiratório na ventilação pulmonar.

\section{MÉTODO}

Após aprovação do Comitê de Ética em Pesquisa da Instituição, foi realizado um estudo na Unidade de Terapia Intensiva Geral Adulto do Hospital Regional de Mato Grosso do Sul em Campo Grande/MS.

Foram incluídos no estudo pacientes adultos de ambos os sexos, com idades entre 16 e 84 anos e submetidos à ventilação mecânica, por via tubo orotraqueal ou traqueostomia, ventilados no modo pressão controlada ou assistida-controlada.

Foram considerados aptos à inclusão, os pacientes cuja indicação ao suporte ventilatório não incluíam doenças obstrutivas de vias aéreas (agudas ou crônicas) ou doenças restritivas prévias. Foram excluídos os pacientes que apresentaram quadro de instabilidade clínica, agitação psicomotora ou alto risco de óbito.

Os responsáveis pelos pacientes assinaram Termo de Consentimento Livre e Esclarecido sobre o protocolo proposto, em conformidade à Declaração de Helsinque.

Para coleta de dados foi utilizado um ventilômetro de Wright da Ferraris acoplado ao ramo expiratório do circuito do ventilador mecânico. Para cada paciente foi mantida a pressão de insuflação, a freqüência respiratória, a pressão positiva término expiratória e a fração inspirada de oxigênio administrados previamente. Com o paciente posicionado em decúbito dorsal em aproximadamente $30^{\circ}$ de elevação do tronco, foi realizado ajuste do tempo inspiratório em $1 \mathrm{seg}$, incrementando em 0,2 seg, até o valor limite de 1,6 seg.

Cada ajuste do tempo inspiratório foi mantido por um minuto antes da coleta de dados, como forma de permitir adaptação do sistema ventilatório ao parâmetro regulado. A seguir foi realizada a medição do volume-corrente e volume-minuto expirados através do destravamento do ventilômetro, previamente instalado no circuito.

O valor do volume-minuto foi obtido através do registro de todo volume expirado no intervalo de um minuto.

Foi considerado para registro, o volume-corrente médio obtido do quociente entre volume-minuto e a freqüência respiratória.

Este procedimento de coleta foi aplicado a cada ajuste de tempo inspiratório e os dados coletados foram registrados em ficha pré-elaborada para posterior análise.

Os valores coletados foram tratados estatisticamente pelo teste de Friedman, observando nível significativo de $p<0,05$.

\section{RESULTADOS}

Foram avaliados o volume-corrente $\left(\mathrm{V}_{\mathrm{T}}\right)$ e o volumeminuto $\left(\mathrm{V}_{\mathrm{E}}\right)$ de 13 pacientes nos tempos inspiratórios de $1 ; 1,2 ; 1,4$ e 1,6 seg. Na média dos $V_{T}$ observouse um aumento crescente pós-incremento do tempo inspiratório. Encontrou-se aumento significativo $(\mathrm{p}<$ $0,05)$ na comparação dos $V_{T}$ médios nos tempo inspiratórios entre 1 e 1,6 seg e de 1,2 e 1,6 seg (Figura 1).

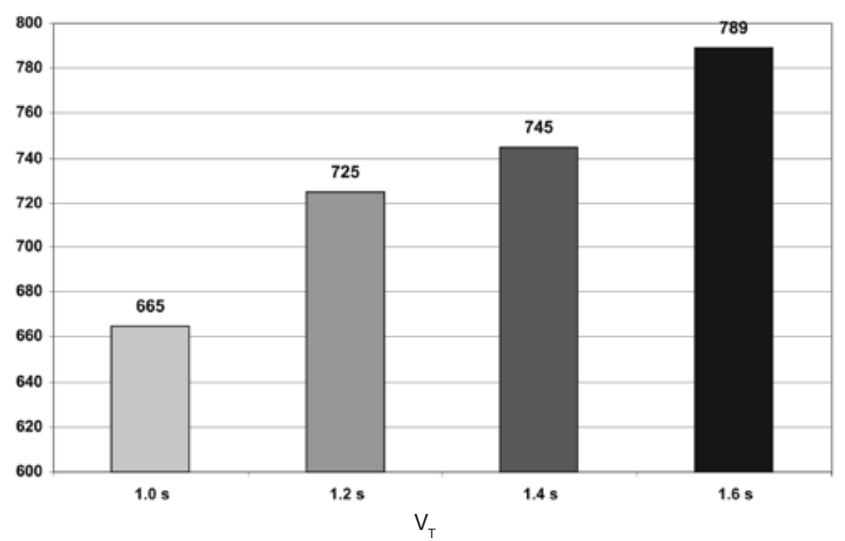

Figura 1 - Comparação das Médias do Volume-Corrente $\left(\mathrm{V}_{\mathrm{T}}\right)$

Três pacientes apresentaram $\mathrm{V}_{\mathrm{T}}$ no tempo inspiratório de 1 seg maior que no tempo de 1,2 seg, contrariando o observado na média. Um paciente apresentou o $V_{T}$ 
(524 mL) no tempo inspiratório de 1,2 seg maior que no tempo de $1,4 \mathrm{seg}(481,5 \mathrm{~mL})$. Dois pacientes apresentaram $\mathrm{V}_{\mathrm{T}}$ no tempo de 1,4 seg maior que no tempo inspiratório de 1,6 seg (Tabela 1).

Tabela 1 - Volume-Corrente de acordo com o Tempo Inspiratório Ajustado

\begin{tabular}{lcccc}
\hline & \multicolumn{5}{c}{$\mathrm{V}_{\mathrm{T}}$} \\
\hline Pacientes & $1 \mathrm{~s}$ & $1,2 \mathrm{~s}$ & $1,4 \mathrm{~s}$ & $1,6 \mathrm{~s}$ \\
\hline 1 & 590 & 730 & 780 & 700 \\
2 & 487 & 524 & 482 & 511 \\
3 & 750 & 810 & 810 & 860 \\
4 & 1040 & 1240 & 1305 & 1380 \\
5 & 750 & 700 & 700 & 820 \\
6 & 782 & 856 & 862 & 941 \\
7 & 720 & 870 & 900 & 960 \\
8 & 813 & 705 & 740 & 840 \\
9 & 415 & 385 & 415 & 406 \\
10 & 530 & 610 & 660 & 710 \\
11 & 670 & 730 & 730 & 750 \\
12 & 650 & 730 & 750 & 802 \\
13 & 450 & 534 & 553 & 573 \\
Média & 665,1538 & 724,9231 & 745,1154 & 788,6923 \\
DP & 172,7719 & 207,6541 & 220,1297 & 240,9545 \\
\hline
\end{tabular}

Em relação ao volume-minuto $\left(V_{E}\right)$, as médias também apresentaram valor crescente no decorrer do incremento do tempo inspiratório, sendo a diferença significativa $(p<0,05)$ do tempo inspiratório de 1 seg para o de 1,6 seg (Figura 2).

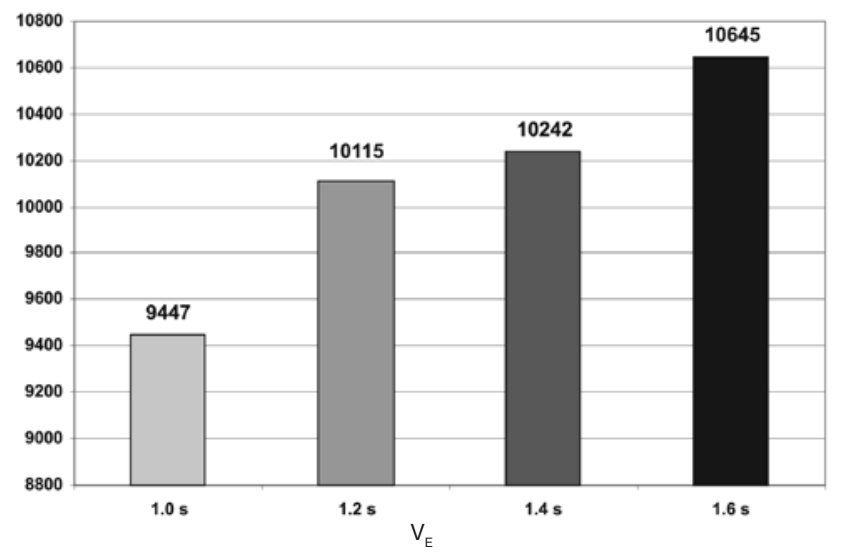

Figura 2 - Comparação das Médias do Volume-Minuto $\left(\mathrm{V}_{\mathrm{E}}\right)$

Três pacientes apresentaram $V_{E}$ maior no tempo inspiratório de $1 \mathrm{seg}$ que no tempo de 1,2 seg. Quatro pacientes apresentaram $V_{E}$ no tempo inspiratório de 1,2 seg maior que no tempo inspiratório de 1,4 seg. Dois pacientes apresentaram os $\mathrm{V}_{\mathrm{E}}$ maiores no tempo inspiratório de 1,4 seg que no tempo de 1,6 seg, como pode ser observado na tabela 2 .

Tabela 2 - Volume-Minuto de acordo com o Tempo Inspiratório Ajustado.

\begin{tabular}{lcccc}
\hline \multicolumn{5}{c}{$\mathrm{V}_{\mathrm{F}}$} \\
\hline Pacientes & $1 \mathrm{~s}$ & $1,2 \mathrm{~s}$ & $1,4 \mathrm{~s}$ & $1,6 \mathrm{~s}$ \\
\hline 1 & 14510 & 13400 & 14370 & 12660 \\
2 & 9740 & 10480 & 9630 & 10220 \\
3 & 11850 & 11870 & 11310 & 12000 \\
4 & 12520 & 14890 & 15660 & 16570 \\
5 & 9840 & 12850 & 13120 & 14110 \\
6 & 8610 & 9420 & 9490 & 10360 \\
7 & 7510 & 8930 & 8030 & 8450 \\
8 & 10580 & 9170 & 9620 & 10920 \\
9 & 6650 & 6150 & 6650 & 6500 \\
10 & 7340 & 8440 & 9260 & 9940 \\
11 & 8250 & 8640 & 8610 & 8760 \\
12 & 8200 & 8700 & 9100 & 9300 \\
13 & 7210 & 8550 & 8300 & 8600 \\
Média & 9446,923 & 10114,62 & 10242,31 & 10645,38 \\
DP & 2364,369 & 2453,221 & 2640,143 & 2667,488 \\
\hline
\end{tabular}

\section{DISCUSSÃO}

Emmerich e col. ${ }^{6}$ relataram que o uso da ventilação por controle de pressão constitui a melhor opção de suporte ventilatório, em termos de otimização da distribuição intrapulmonar do volume-corrente, e apesar de ser amplamente utilizado na prática clínica, não foram encontrados na literatura, dados que correlacionassem o tempo inspiratório com alterações no volume-corrente.

Pode-se observar aumento significativo de volumecorrente no incremento do tempo inspiratório de 1 e 1,2 a 1,6 seg. Laghi e col. ${ }^{9}$ relataram que a importância na manutenção do tempo inspiratório adequado pode garantir o volume de expiração, a freqüência respiratória e a manutenção da abertura de grande parte das unidades alveolares.

Outra vantagem atribuída ao modo ventilatório por controle de pressão é a garantia de respeito aos picos de pressão inspiratórios, característica ausente quando se ventila o paciente por controle de volume. Richard e col. ${ }^{7}$ recomendaram a chamada estratégia de proteção pulmonar, ou seja, manter os alvéolos abertos sem hiperdistendê-los.

Sabe-se que a ventilação mecânica promove profundas alterações no sistema cardiovascular, secundárias à transmissão intratorácica das pressões geradas nas vias aéreas ${ }^{4}$. Neste estudo pode-se observar que indiferente do tempo inspiratório, um volume-corrente ade- 
quado foi gerado, respeitando-se simultaneamente os limites pressóricos intrapulmonares. Emmerich e col. ${ }^{6}$ relataram que a propriedade da ventilação a pressão controlada de garantir uma curva quadrada de pressão durante um tempo determinado, pode representar uma real vantagem na prevenção de lesões induzidas pelo ventilador no caso de lesões pulmonares mais graves. Um fato negativo observado neste estudo foi que, para uma freqüência respiratória de 12 irpm, um aumento no tempo inspiratório de 1 para 1,6 seg, implica em alteração da relação I:E de 1:4 para 1:2,1. O mesmo comportamento pode ser visto quando se trabalhou com freqüência respiratória de 16 irpm, para a qual um incremento análogo causaria uma alteração na relação I:E de 1:2,7 para 1:1,3. Yanos e col. ${ }^{10}$ relataram que o uso de uma relação I:E muito próxima poderia potencializar o risco de algumas importantes complicações, como a diminuição no volume de gás expirado e aumento do risco de barotraumas.

Pinheiro e Carvalho ${ }^{11}$ recomendaram o uso de um volume-minuto em torno de 6 litros. durante o nosso estudo foi observada ocorrência de volumes-minuto significativamente maiores que os valores de referência em vários pacientes, independentemente do tempo inspiratório regulado, sugerindo que nos pacientes avaliados a condução da ventilação mecânica era feita com valores superestimados.

\section{CONCLUSÃO}

Através da análise dos dados obtidos neste estudo pode-se concluir que o incremento do tempo inspiratório na ventilação por controle de pressão pode ter in- fluência na determinação do volume-corrente ofertado ao paciente.

Os métodos de monitorização dos valores de volumecorrente ofertado e o controle gasométrico da geração de volume-minuto adequado, constituem ainda instrumentos essenciais na condução da terapia ventilatória mecânica.

\section{REFERÊNCIAS}

01. Emmerich JC - Suporte Ventilatório: Conceitos Atuais. Rio de Janeiro, $2^{a}$ Ed, Revinter, 1998; 17-36.

02. Stock M, Perel A - Manual de Suporte Ventilatório Mecânico. São Paulo,

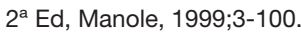

03. Barrera MRR, Ramos FM, Hernandez MIV et al - Empleo de la ventilación mecánica en la unidad de cuidados intensivos. Rev Assoc Mex Med Crit y Ter Int, 2002;16:153-158.

04. Klemm M, Alvarenga J, Fantoni DT et al - Estudo comparativo da ventilação mecânica controlada (VMC) com ou sem o emprego da pressão positiva no final da expiração (PEEP) sobre a variação da pressão interpleural em anestesia eqüina. Braz J Vet Res Anim Sci, 1998;35:260-265.

05. Villagra A, Ochagavia A, Vatua $S$ et al - Recruitment maneuvers during lung protective ventilation in acute respiratory distress syndrome. Am J Res Crit Care Med, 2002;165:165-170.

06. Emmerich JC, Siqueira, HR, Gomes AS et al - Estudo comparativo e retrospectivo: ventilação ciclada a volume com fluxo desacelerado, ventilação por controle de pressão e ventilação com pressão de suporte e volume assegurado aplicadas de maneira seqüencial em pacientes com lesão pulmonar aguda de intensidade moderada à grave. RBTI, 1998;10:84-89.

07. Richard JC, Maggiore SM, Jonson B et al - Influence of tidal volume on alveolar recruitment. Respective role of PEEP and a recruitment maneuver. Am J Respir Crit Care Med, 2001;163:1609-1613.

08. Brower RG, Ware LB, Berthiaume $Y$ et al - Treatment of ARDS. Chest, 2001;120:1347-1367.

09. Laghi F, SegaL J, Choe WK et al - Effect of imposed inflation time on respiratory frequency and hyperinflation in patients with chronic obstructive pulmonary disease. Am J Respir Crit Care Med, 2001;163:1365-1370.

10. Yanos J, Watling SM, Verhey J - The physiologic effects of inverse ratio ventilation. Chest, 1998;114:834-838.

11. Pinheiro CTS, Carvalho WB - Programa de Atualização em Medicina Intensiva. AMIB. Porto Alegre, Artmed/Editora Médica Panamericana, 2003;49-52. 